\title{
The Nicotinic Blocking Agents $\alpha$-Tubocurare and $\alpha$-Bungarotoxin Save Motoneurons from Naturally Occurring Death in the Absence of Neuromuscular Blockade
}

\author{
Freya Hory-Lee and Eric Frank \\ Department of Neurobiology, University of Pittsburgh Medical School, Pittsburgh, Pennsylvania 15261
}

\begin{abstract}
Motoneurons undergo a phase of target-dependent cell death during development. In chick embryos, motoneuronal death is blocked by the application of the nicotinic antagonists $\alpha$-tubocurare (dTC) or $\alpha$-bungarotoxin ( $\alpha$-BTX). Paralytic doses of these drugs also increase intramuscular nerve branch formation. To investigate the possibility that a neuronal rather than a muscle-type nicotinic $A C h$ receptor (nAChR) might be responsible for the toxin-induced arrest of naturally occurring motoneuronal death, we compared the doses of dTC and $\alpha$-BTX required for paralysis with those needed to protect motoneurons from cell death. We also measured the effects of dTC on the survival of retrogradely labeled motoneurons in culture, and of various doses of dTC on intramuscular nerve branch formation. Subparalytic doses of dTC caused small but significant increases in nerve branch number, while higher doses produced larger effects. In contrast, motoneuronal survival was already maximal at doses of dTC or $\alpha-B T X$ below those needed for a visible effect on limb movement. Moreover, dTC increased motoneuron survival in culture, in the absence of muscle cells and muscle-type nAChRs. Nicotinic blocking agents can therefore rescue motoneurons with minimal depression of neuromuscular transmission, suggesting that this effect may be mediated through neuronal, rather than muscle-type, nAChRs.
\end{abstract}

[Key words: motoneurons, naturally occurring cell death, d-tubocurare, $\alpha$-bungarotoxin, neuronal nicotinic ACh receptors, intramuscular nerve branch formation]

Motoneurons are among the earliest neurons born in the vertebrate spinal cord, and among the earliest neurons to send axons out into the developing vertebrate limb (Hamburger, 1948; Landmesser, 1978). During the period of synaptogenesis with the developing muscle fibcrs, motoncurons then go through a pcriod of naturally occurring death (Hamburger, 1975). The survival of motoneurons during this period is target dependent, mediated by some trophic substance or substances produced by developing

\footnotetext{
Received Feb. 2, 1995; revised May 16, 1995; accepted May 18, 1995.

We thank Dr. Zeljka Korade for valuable technical advice and Ms. Xiaoping Chen for valuable technical support. This work was supported by RO1NS24373 from the National Institutes of Health to E.F.; F.H.L. was supported by 5 T32 HD7343-04 from the Department of Health and Human Services Public Health Service.

Correspondence should be addressed to Freya Hory-Lee and Eric Frank, Department of Neurobiology, University of Pittsburgh Medical School, 3550 Terrace Street, Pittsburgh, PA 15261.

Copyright @ 1995 Society for Neuroscience $0270-6474 / 95 / 156453-08 \$ 05.00 / 0$
}

muscles (Oppenheim et al., 1993). The identity of these substances, however, remains unknown.

Essentially all motoneurons gencrated in the chick spinal cord survive the period of naturally occurring death if a paralytic dose of either $\alpha$ - bungarotoxin ( $\alpha$-BTX) or $d$-tubocurare (dTC) is applied to developing chick embryos throughout this period (Pittman and Oppenheim, 1978; Pittman and Oppenheim, 1979). The interpretation has been that a functional neuromuscular interaction is necessary for the regulation of naturally occurring motoneuron dealh, because cholinergic neuromuscular blockade results in increased motoneuron survival. In addition to blocking the neuromuscular junction, however, these cholinergic drugs also can act on nicotinic $\mathrm{ACh}$ receptors (nAChRs) on neurons. There are two general classes of neuronal nAChR, those that bind $\alpha$-BTX and those that do not. Both classes are distinct from nAChRs found in skeletal muscle or fish electric organs (Lindstrom et al., 1991). Although the properties of neuronal nAChRs that bind $\alpha$-BTX are only beginning to be characterized, they appear to mediate a variety of functions not normally associated with ligand-gated channels that mediate synaptic transmission (for example, see Vijayaraghavan et al., 1992).

A specific binding site for $\alpha$-BTX in the developing chick spinal cord has recently been reported, and exogenous ${ }^{125} \mathrm{I} \alpha-$ BTX administered to chick embryos on embryonic day (E)6 has been found to bind to nicotine-sensitive sites in the spinal cord by E7 (Renshaw et al., 1993). As a number of neuronal nAChRs with a variety of functions have been identified, and as there is a specific binding site for $\alpha$-BTX in the developing chick spinal cord, it seemed reasonable to consider the possibility that some site other than the neuromuscular junction is responsible for nicotinic antagonist-mediated arrest of motoneuron death. In this study, we show that levels of dTC or $\alpha$-BTX below those producing visible neuromuscular blockade are still sufficient to produce substantial increases in motoneuronal survival in chick embryos. In addition, we find that dTC can act on motoneurons in culture in the absence of muscle cells, and therefore in the absence of a neuromuscular junction and muscle nAChRs. Finally, as paralytic doses of dTC increase intramuscular nerve branching as well as rescuing motoneurons from cell death, increased branching has been postulated to be crucial for increased motoneuron survival (Dahm and Landmesser, 1988). We find that subparalytic doses of dTC sufficient to save motoneurons do result in small increases in branch number, but the relationship between branch number and motoneuron survival is not linear.

\section{Materials and Methods}

Drug treatments in ovo. Drugs wcre dissolved in chick Ringer's solution, and applied daily to the chorioallantoic membrane of White Leg- 
horn chick embryos from E5 to E10, in a total volume of 200-400 $\mu \mathrm{l}$. Chick Ringer's solution consisted of $154 \mathrm{mM} \mathrm{NaCl}, 5.6 \mathrm{~mm} \mathrm{KCl}$, and $2.4 \mathrm{mM} \mathrm{CaCl}_{2}$. Control animals were treated with Ringer's solution without drugs. In animals treated with nicotinic blockers, the dose necessary to maintain embryonic paralysis changes during the treatment period (Pittman and Oppenheim, 1979). The paralytic doses of dTC (Sigma) were $2 \mathrm{mg}$ daily on E5-7, $3 \mathrm{mg}$ daily on E8-9, and $4 \mathrm{mg}$ on E10. The paralytic doses of $\alpha$-BTX (Sigma) were $100 \mu \mathrm{g}$ on E5-7, 75 $\mu \mathrm{g}$ on E8-9, and $50 \mu \mathrm{g}$ on E10. In this report, other doses of these drugs are subsequently expressed as percentages of the paralytic dose. Doses of dTC varied from $2.5 \%$ to $100 \%$, while doses of $\alpha$-BTX varied from $1.2 \%$ to $100 \%$. Between drug applications, eggs were maintained in an incubator at $37^{\circ} \mathrm{C}$ and approximately $60 \%$ humidity.

The number of hindlimb movements in each embryo from E7 to E10 was counted in a $2 \mathrm{~min}$ observation period, beginning approximately 2 $\mathrm{hr}$ after drug application. In addition, movements were counted in the embryos treated with paralytic doses of dTC or $\alpha$-BTX from E7 to E11 just prior to the following drug application. In this way it was possible to be certain that animals given paralytic doses of a drug were in fact immobile for the duration of the drug treatment. In contrast, animals given a dose of drug determined to be nonparalytic were fully mobile for the majority of each $24 \mathrm{hr}$ period. The data for E9 was subsequently used to compare the effects of the different doses of the drugs on motility.

On E11, surviving embryos were sacrificed and the spinal cords with adjacent dorsal root ganglia (DRGs) were dissected and fixed overnight in $4 \%$ paraformaldehyde. The cords were then dehydrated in ethanol followed by xylene, embedded in paraffin, and serially sectioned at 10 $\mu \mathrm{m}$. Sections were stained with cresyl violet, and the number of motoneurons in sections throughout the lumbar spinal cord were counted. All sections were initially examined to define the boundaries of lumbar segments 1 to 5 (L1-L5). The boundary of a segment was defined as the midpoint between the rostral and caudal borders of adjacent DRGs. Thoracic segment 12 (T12) was identified by the presence of the last rib and the small size of the last thoracic DRG relative to the first lumbar DRG. Motoneurons were then counted in every fifth section from L1 through L5. These cells were identified by their large size, dense staining and location in the lateral motor column (LMC). Only large cells in the LMC containing at least one nucleolus were counted as motoneurons (Hamburger, 1975; Pittman and Oppenheim, 1979; Williams et al., 1987).

Motoneuron survival for animals in each experimental group was assessed by determining the average number of motoneurons per $10 \mu \mathrm{m}$ section in the L4 region. This method was chosen over the more common method of estimating the total number of motoneurons per seg. ment. The assignment of segment boundaries was sometimes difficult because DRGs can become damaged or pulled out of alignment during processing. An "incorrect" boundary assignment will affect the total number of motoneurons determined to be in a segment, but will have less effect on the average number of motoneurons per section within a segment. The L4 region was selected for comparing different treatment groups because the density of motoneurons in the lumbar region increases from $\mathrm{L} 1$ through the start of $\mathrm{L} 3$ and then plateaus from the end of L3 through L5 (Williams et al., 1987). L4 is therefore in the center of the plateau region, which means that a small error in the assignment of segment boundaries is unlikely to result in a significant error in the determination of motoneuron density (motoneurons per section). In most cases motoneurons were counted on both left and right sides of the spinal cord and the numbers were averaged.

Embryos kept until E16 were treated daily from E5 to E10 with either Ringer's solution, paralytic doses of dTC, or 10\%-paralytic doses of dTC as described previously. Half the embryos in the $10 \%$ treatment group then received $0.4 \mathrm{mg}$ of dTC from E1 $1-12$ and $0.5 \mathrm{mg}$ of dTC from E13-15. All other embryos were treated with Ringer's solution from E11-15. (It was not possible to continue treatment of embryos with a paralytic dose of dTC from E11-15 because of very high mortality rates.) On E16, embryos were sacrificed, the spinal cord was processed, and motoneurons were counted in every tenth section throughout the lumbar spinal cord.

In embryos with limb ablations, a hindlimb bud was removed on E2 with tungsten needles. The egg was then resealed and returned to the incubator until E5. Beginning on E5 and continuing through E10, embryos were treated with drugs as described previously. In addition to paralytic doses, $5 \%$-paralytic doses of dTC and $\alpha$-BTX were used. Control animals were treated with chick Ringer's solution. Animals were
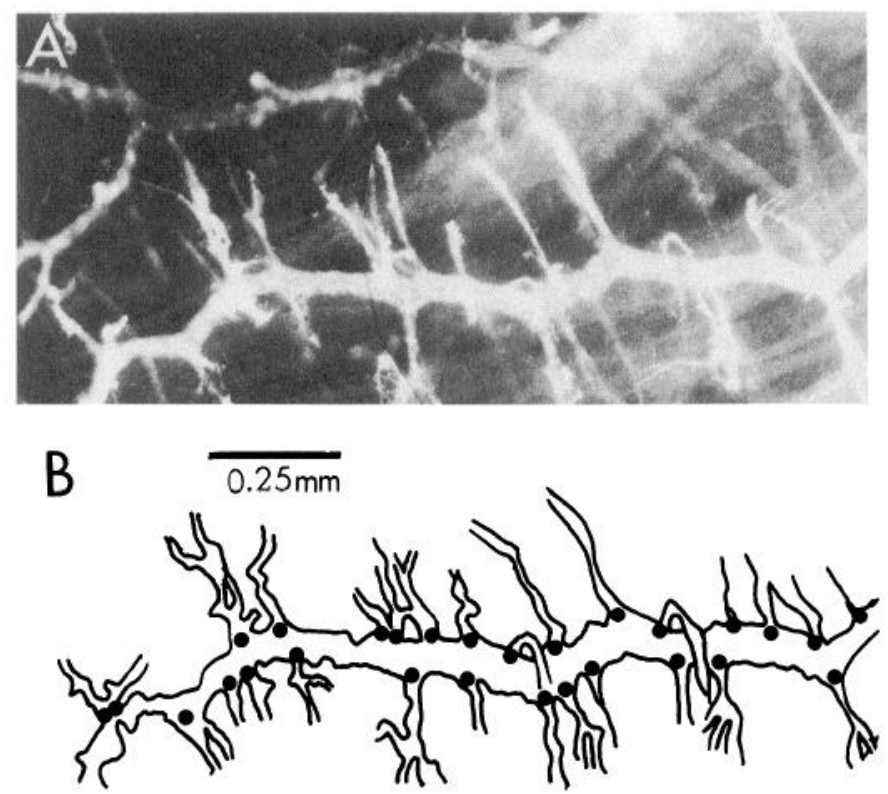

Figure 1. Portion of a whole mount of the iliofibularis muscle from an embryo treated with paralytic dTC after staining to visualize the intramuscular nerves. A, Section of anterior nerve trunk from the slow region of the muscle. $B$, Drawing of the same nerve trunk with dots marking the side branches.

sacrificed on E11, and the spinal cord was processed and motoneurons counted. The number of motoneurons on the limb-ablated and control sides were recorded separately for comparison with each other.

Intramuscular nerve branching patterns. Muscle nerve branching patterns were assessed using a procedure based on that reported by Dahm and Landmesser (1988). Chick embryos were treated from E5 to E10 with a paralytic dose of dTC, a 5\%-paralytic dose, a 10\%-paralytic dose, or chick Ringer's solution. On E11 the embryos were taken from the eggs, and the skin and connective tissue were removed from both hindlimbs to reveal the underlying musculature. The iliofibularis muscles were identified and left intact, while the remaining muscles, connective tissue and main nerve trunks were stripped away. The iliofibularis muscles were then fixed in ice-cold acetone for $2 \mathrm{~min}$, washed $3 \times 20 \mathrm{~min}$ each in phosphate-buffered saline (PBS) and incubated overnight in undiluted $7 \mathrm{G} 4$ hybridoma supernatant with $0.02 \%$ Tween20 . The monoclonal antibody $7 \mathrm{G} 4$ recognizes chicken neurofilaments, and stains intramuscular nerve tracts (E.F., unpublished observations). The muscles were washed $9 \times 10$ min each in PBS with $0.05 \%$ Tween20 , fixed in $4 \%$ paraformaldehyde for $30 \mathrm{~min}$, and washed another $3 \times$ $20 \mathrm{~min}$ each in PBS with $0.05 \%$ Tween-20. The muscles were then incubated overnight at $4^{\circ} \mathrm{C}$ in blocking buffer containing the fluoresceincoupled secondary antibody goat anti-mouse IgM 1211-0201 (Cappel). Blocking buffer consisted of $10 \%$ horse serum and $1 \%$ bovine serum albumin in PBS with $0.02 \%$ Tween-20. Finally, the muscles were washed $9 \times 10$ min each in PBS with $0.05 \%$ Tween-20, removed from the limb, and mounted between two coverslips in Mowiol (Cal Biochem).

Collateral branching of the nerve trunks in the slow anterior region of the iliofibularis muscle forms regularly spaced side branches in a stereotyped manner (Dahm and Landmesser, 1988). Fluorescence photographic montages were constructed of the anterior region of each muscle so that the branching patterns throughout this region could be visualized. The number of side branches on the anterior intramuscular nerve trunks were counted directly from these montages, as is shown in Figure 1. For purposes of control and comparison, embryonic motility was measured in these animals, and motoneuron survival was assessed for a subset of three animals from each treatment group using the methods described previously. The values were not found to differ from the animals used in the initial dose-response determinations, and these data were therefore pooled with the earlier results.

Retrogradely labeled dissociated motoneuron cultures. Motoneurons from E4 or E5 embryos were retrogradely labeled with either an aqueous suspension of green fluorescent latex microspheres (Lumaflour, Inc.) 
or an ethanol solution $(12.5 \mathrm{mg} / \mathrm{ml})$ of the fluorescent dye $1,1^{\prime}$-dioctadecyl-3,3,3',3',-tetramethyl-indocarbo-cyanine perchlorate (diI) pressure-injected into the leg muscles through a micropipette with a tip diameter of approximately $100 \mu \mathrm{m}$. Following injections, eggs were sealed and returned to the incubator. After $22-24 \mathrm{hr}$, the lumbar spinal cords were removed, dissociated (Tanaka, 1987), and enriched for neurons (Wakade et al., 1982). Droplets of the single cell suspension $(8 \mu \mathrm{l}$, each containing approximately 15,000 cells) were then preplated onto the bottom of $1 \mathrm{~cm}$ well multidishes (Nunc) coated with polyornithine and laminin. The dishes were kept at $37^{\circ} \mathrm{C}$ for $1 \mathrm{hr}$, following which the initial growth medium was added to the wells. The initial growth medium consisted of Minimal Essential Medium (GIBCO) supplemented with $10 \%$ NuSerum (Collaborative Research Inc.), $2 \mathrm{~mm} \mathrm{L-}$ glutamine, and $1 \%$ antibiotic-antimycotic (GIBCO). For some cultures it also contained $25 \mu \mathrm{g}$ of muscle extract per milliliter and/or $10 \mu \mathrm{M}$ dTC. Muscle extract was prepared according to Davies (1987).

In each experiment, each condition was replicated six to eight times. Different cultures were given medium enriched with muscle extract and/or dTC. Control cultures were given neither additive. Labeled cells were counted in living cultures with fluorescence optics using a $20 \times$ objective on an inverted microscope after 24 and $48 \mathrm{hr}$. Initially, the entire colony was scanned to check for a uniform density of labeled cells. Subsequently, a representative field with an area of $0.79 \mathrm{~mm}^{2}$ was selected, and the number of fluorescent cells within that field was recorded. An example of a spinal cord culture containing retrogradely labeled motoneurons is shown in Figure 2. To determine if the effect of dTC on motoneuron survival in spinal cord cultures was specific, rather than a general effect on all neurons, we repeated the experiments using retrogradely labeled muscle sensory neurons (prepared as previously described; Hory-Lee et al., 1993), instead of motoneurons.

In all experiments described in this article, the two-tailed $t$ test for paired samples was used for statistical analysis.

\section{Results}

\section{Drug treatments in ovo}

The possibility that nicotinic cholinergic blockers may save motoneurons by acting directly on neuronal receptors was investigated by comparing dose-response curves for embryonic paralysis with dose-response curves for motoneuron survival. If increased motoneuron survival and paralysis were found to be produced by very different doses of dTC or $\alpha$-BTX, this would argue that different populations of receptors might be mediating the two effects.

The results of the experiments with dTC were that substantially lower doses of drug than those resulting in paralysis were still effective in saving motoneurons during the period of targetdependent cell death. Doses of dTC corresponding to $5 \%$ and $10 \%$ of the paralytic dose (each dose of drug is subsequently expressed as a percentage of the paralytic dose) did not change the frequency of hindlimb movements throughout the treatment period, but still resulted in a maximal effect on motoneuron survival. The dose-response curves demonstrating the effects of dTC on motoneuron survival and embryonic motility are shown in Figure 3.

A potential weakness in the use of dTC for these experiments is that it is not highly specific for cholinergic receptors (Katz and Miladi, 1978). The dose-response experiments were therefore repeated with $\alpha$-BTX, which is a more specific nicotinic antagonist (Bulgar et al., 1977). The effects of lower doses of $\alpha$-BTX on both embryonic motility and motoneuron survival were similar to the effects of lower doses of dTC. A dose of 5\% was sufficient to rescue all motoneurons from death, but had no observable effect on embryonic motility. These results, demonstrating the effects of $\alpha$-BTX on motoneuron survival and embryonic movement, are shown in Figure 4. As subparalytic doses of $\alpha$ - BTX were effective at preventing the loss of virtually all motoneurons, it is probable that both dTC and $\alpha$-BTX act at the
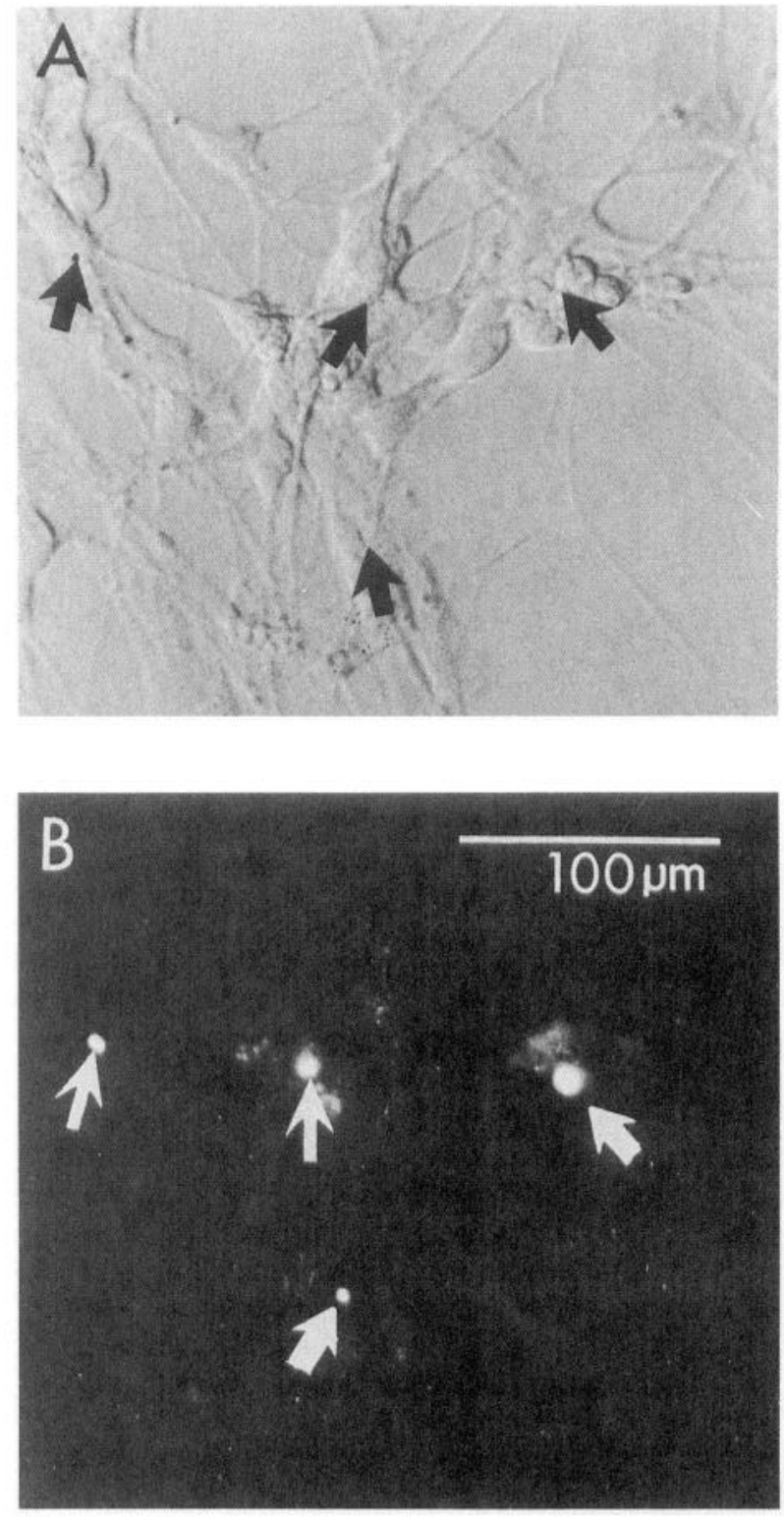

Figure 2. Retrogradely labeled motoneurons in dissociated spinal cord cultures after $48 \mathrm{hr}$ in medium containing muscle extract, viewed with phase-contrast $(A)$ and fluorescein $(B)$ optics. Arrows indicate labeled cells. To show detail, the area in the photograph is smaller than the representative microscope field area of $0.79 \mathrm{~mm}^{2}$ used to record numbers of fluorescent cells.

same (nicotinic cholinergic) site to block target-dependent motoneuron death.

Not only was the frequency of hindlimb movement unaffected by the subparalytic doses of dTC or $\alpha$-BTX still sufficient to rescue motoneurons, but there also was no qualitative difference between movement in treated versus control animals. A dose of dTC five times lower than the minimum dose that caused some paralysis (5\% vs $25 \%$; refer to Fig. 3) was still maximally effective in saving motoneurons. The fact that these concentrations of dTC and $\alpha$-BTX do not affect visibly movement not only argues against a major involvement of the neuromuscular junction in naturally occurring motoneuron death, it also argues against involvement of motoneuronal activity in the CNS. Anything that affected the activity of motoneurons would indirectly 


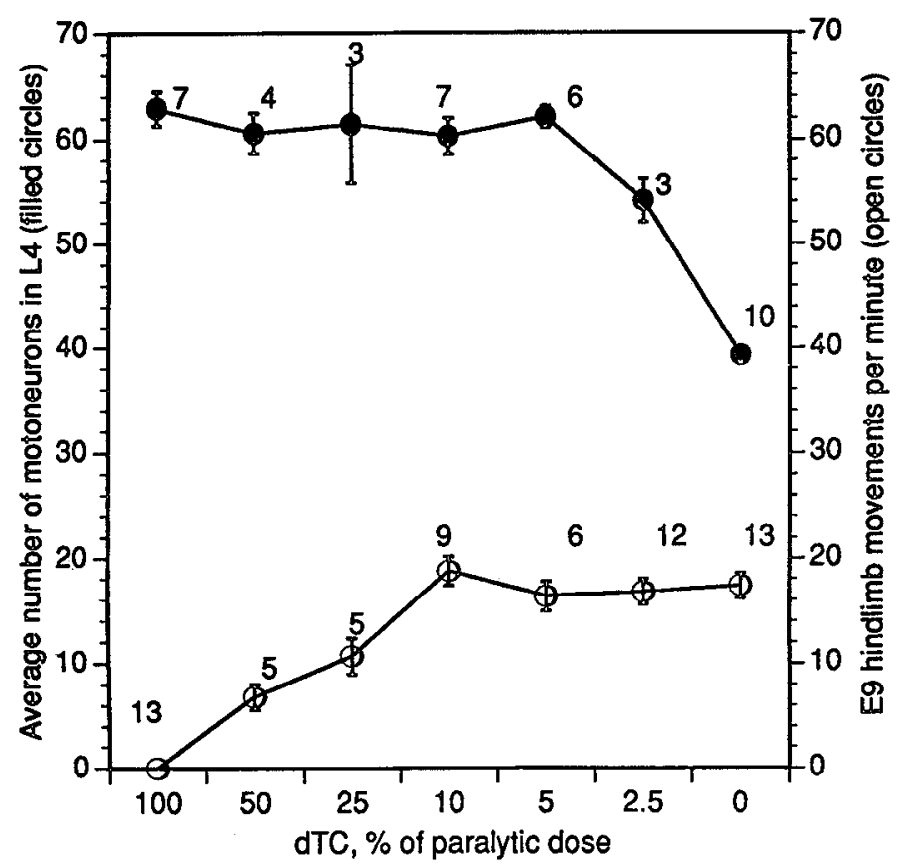

Figure 3. Dose-dependent effects of dTC on motoneuronal survival and embryonic motility. Each point indicates the average number of motoneurons \pm SEM per $10 \mu \mathrm{m}$ section in the L4 region of the spinal cord on E11 (O), or the average number of hindlimb movements per minute \pm SEM on E9 approximately $2 \mathrm{hr}$ after drug application $(\mathrm{O})$. The doses of dTC recorded along the horizontal axis are shown as percentages of the paralytic dose and therefore decrease from $100 \%$; $0 \%$ represents injections of chick Ringer's solution. The number of embryos studied is indicated next to each point. All doses of dTC less than $100 \%$ resulted in significantly more hindlimb movements $(p<$ $0.01)$ than the paralytic dose. In addition, there was no significant difference in movement frequency between doses of dTC of $\leq 10 \%$ and control. Only the doses of $2.5 \%$ and $0 \%$ (control) resulted in significantly fewer motoneurons than the paralytic dose $(p<0.01)$.

affect motility. This may mean that the nAChRs involved in blocking motoneuron death play a role unrelated to nicotinic cholinergic synaptic transmission.

Limb bud ablation resulted in massive motoneuron death even when embryos were treated with cholinergic blocking agents. These results are presented in Table 1 . The numbers of motoncurons on the unablated side were normal in control animals treated with chick Ringer's solution and high, as a result of toxin-induced arrest of naturally occurring motoneuron death, in animals treated with dTC or $\alpha$-BTX. In contrast, very low numbers of motoneurons were present on the ablated side of spinal cords from all treatment groups. Two embryos were treated with a paralytic dose and two embryos were treated with a subparalytic dose of dTC. For $\alpha$-BTX, one embryo was treated with a paralytic dose, and two embryos were treated with a subparalytic dose. The subparalytic dose in each case was $5 \%$. There was no difference between the results obtained with paralytic and subparalytic doses, so the data have been pooled in Table 1. These results are in agreement with a previous report (Pittman and Oppenheim, 1979) that dTC treatment does not rescue motoneurons in limb-ablated embryos.

A distinction has been made between treatments that support motoneuron survival throughout the time of naturally occurring motoneuron death and treatments which continue to support motoneurons after this period (Pittman and Oppenheim, 1979). Motoneuronal survival on E16 was therefore examined for different

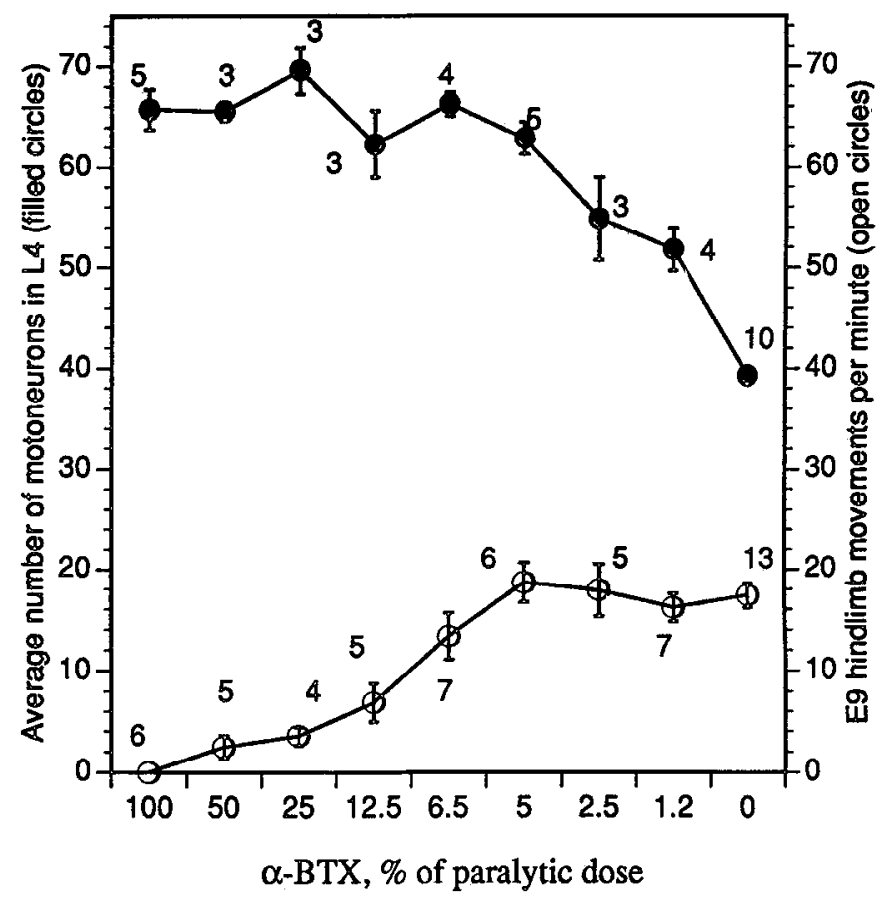

Figure 4. Dose-dependent effects of $\alpha$-BTX on motoneuronal survival (O) and embryonic motility $(O)$. All measurements were made as in Figure 3. Doses of $\alpha-\mathrm{BTX} \leq 6.5 \%$ resulted in significantly more hindlimb movements $(p<0.01)$ than the paralytic dosc. In addition, there was no significant difference in movement frequency between doses of dTC of $\leq 6.5 \%$ and control. Only the doses $1.2 \%$ and $0 \%$ (control) resulted in significantly fewer motoneurons than the paralytic dose $(p$ $<0.01$ )

treatment groups. The results are presented in Table 2. Survival in embryos with paralytic dTC administered through E10 remained high on E16, while in embryos with only a $10 \%$ dose through E10 motoneuron number declined to control levels by E16. If, however, embryos were given the lower dose of dTC through E15, survival remained high on E16. The paralytic dose of drug given through E10 probably has a persistent effect, relative to the subparalytic dose, simply because subparalytic amounts of the drug remain in the egg throughout the later time period.

\section{Drug treatments in vitro}

The limb ablation experiments imply that treatment of embryos with nicotinic blockers does not obviate the requirement of motoneurons for their target muscles. One interpretation is that mo-

Table 1. The effects dTC and $\alpha$-BTX on motoneuron survival in embryos in which one hind limb-bud was ablated on E2

\begin{tabular}{llll} 
& & \multicolumn{2}{l}{ Number of motoneurons } \\
\cline { 3 - 4 } Treatment & $n$ & Control & Limb-bud ablation \\
\hline dTC & 4 & $69.1 \pm 2.8$ & $11.1 \pm 2.6$ \\
$\alpha-B T X$ & 3 & $62.8 \pm 2.5$ & $11.6 \pm 4.2$ \\
Ringer's & 3 & $38.2 \pm 3.0$ & $10 \pm 3.5$
\end{tabular}

Motoneurons were counted on both the nonablated (control) and experimental sides; the figures are the average number of motoneurons ( \pm SEM) per $10 \mu \mathrm{m}$ section in the L4 region of the spinal cord. Chick Ringer's solution was used as a control injection. Drug-treated embryos were given a dose of $100 \%$ or $5 \%$. There was no difference between the results obtained with these two doses, so the data were pooled. 
Table 2. The effects of dTC treatment on motoneuronal survival by E16

\begin{tabular}{lll} 
Treatment & $n$ & $\begin{array}{l}\text { Number of } \\
\text { motoneurons }\end{array}$ \\
\hline $100 \%$ dTC to E10 & 3 & $49.4 \pm 4.5$ \\
$10 \%$ dTC to E10 & 2 & 38 \\
$10 \%$ dTC to E15 & 4 & $53.3 \pm 3.7$ \\
Chick Ringer's & 2 & 30.5
\end{tabular}

Counts are as in Table 1. Chick Ringer's solution was used as a control injec tion. Drug-treated embryos were given a dose of $100 \%$ or $10 \%$ through E10. Embryos given the $100 \%$ dose, and half the embryos given the $10 \%$ dose were then treated with chick Ringer's solution from E11-E 15 . The remaining embryos with the $10 \%$ dose continued to be treated with that dose until E15.

toneurons require target-derived trophic factors even when treated with nicotinic blockers. Alternatively, however, the nicotinic blockers might act directly on the neuromuscular junction. Limb movement is only a crude assay of neuromuscular activity, and it is possible that the subparalytic doses of the nicotinic blockers that rescue motoneurons do decrease activity, although not enough to visibly alter motility. To test whether nicotinic cholinergic receptor blockade was still effective in the absence of muscle nAChRs, we studied whether dTC increased the survival of identified motoneurons in mixed spinal cell cultures grown with and without muscle extract. The effect of dTC on the survival of retrogradely labeled muscle sensory neurons was also examined as a control for the specificity of the effect on motoneurons.

The survival of retrogradely labeled motoneurons in spinal cord cultures plated with muscle extract was potentiated by dTC. In contrast, the presence of dTC in DRG cultures did not affect the survival of muscle sensory neurons, even in medium containing muscle extract. The effects of these treatments on sensory and motor neurons are compared in Figure 5. After $48 \mathrm{hr}$ in culture, motoneuron survival with muscle extract plus dTC was approximately 1.5 times greater than survival in muscle extract alone. The survival of motoneurons in dTC without muscle extract was marginally better than survival in control cultures which were grown in medium supplemented only with $\mathrm{Nu}-$ Scrum, but the improvement was not statistically significant.

There was no improvement in the survival of muscle sensory neurons with dTC; the effect of dTC on survival appears therefore to be specific to motoneurons. The results of these experiments with a nicotinic blocking agent in vitro indicate that dTC potentiated the survival effects of muscle extract rather than affecting motoneuron survival independently. That is, even in the presence of the nicotinic blocker, motoneurons required some soluble muscle-derived molecule(s). Consistent with this, dTC was also found to be ineffective in protecting motoneurons in culture from degeneration following the withdrawal of muscle extract (data not shown). These results are also consistent with the observation that motoneurons die in limb-ablated animals even when the animals are treated with dTC. Interestingly, however, this effect does not require the presence of muscle cells and hence, neuromuscular junctions. Nicotinic blockade can apparently potentiate the effect of muscle-derived factors on motoneurons by acting directly on spinal cord cells, presumably on the motoneurons themselves.

\section{Intramuscular nerve branching patterns}

A variation of the neurotrophic hypothesis commonly believed to apply to developing motoneurons is that muscle fibers pro-

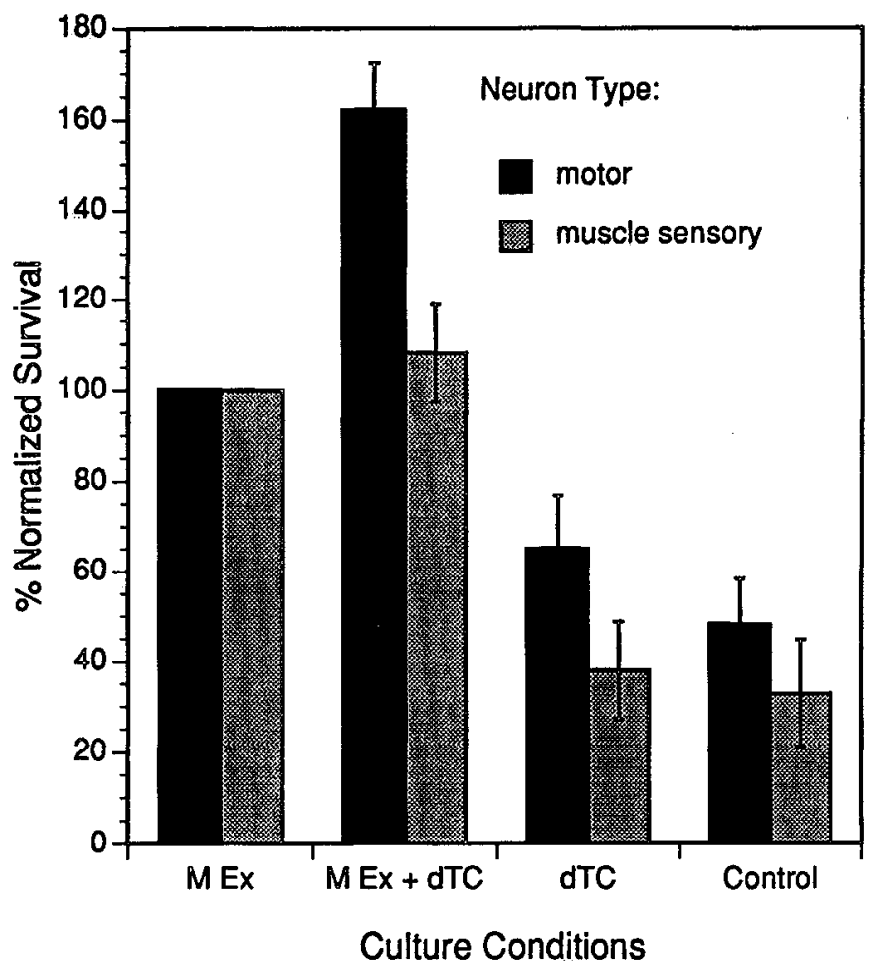

Figure 5. Survival of retrogradely labeled motoneurons and muscle sensory neurons in culture in the presence of muscle extract $(M E x)$ and/or dTC. Control cultures were grown without muscle extract or dTC. Each bar value ( \pm SEM) represents an average of five separate experiments, in which each condition was replicated six to eight times. Labeled neurons were counted after $48 \mathrm{hr}$ in culture, as described in Materials and Methods, and counts were normalized to $100 \%$ for the value in muscle extract. Motoneuronal survival in cultures with muscle extract plus dTC as compared to muscle extract alone was significantly greater $(p<0.025)$. The survival of motoneurons in dTC without muscle extract was marginally better than survival in control cultures which were grown in medium supplemented only with NuSerum, but the improvement was not statistically significant $(p<0.3)$. The survival of muscle sensory neurons in muscle extract was not affected by the presence of dTC.

duce enough trophic factor for all motoneurons, but that neuronal access to the factor is limited in some way (Oppenheim, 1989; Dahm and Landmesser, 1991). Nicotinic blockers do not upregulate the production of muscle-derived trophic factors (Tanaka, 1987; Houenou et al., 1991), so perhaps they increase motoneuronal access to these factors. This hypothesis is supported by the observation that treatment with paralytic doses of dTC or $\alpha$-BTX greatly enhances intramuscular nerve branching, and results in increased synaptogenesis between motor axons and muscle fibers (Dahm and Landmesser, 1988; Dahm and Landmesser, 1991). Nonparalytic doses of dTC also rescue motoneurons from naturally occurring death. It was therefore interesting to determine if these lower doses also increase intramuscular nerve branching.

Paralytic doses of dTC are reported to increase the number of side branches in the slow region of the iliofibularis muscle approximately twofold (Dahm and Landmesser, 1988). The result of paralytic dTC treatment determined here was essentially the same, resulting in a significant incrcase from $9.6 \pm 0.5$ to 19.1 \pm 1.0 branches per mm of nerve trunk $(p<0.01)$. There was not, however, a linear correlation between the effects of different doses on side branch formation and either embryonic motility 


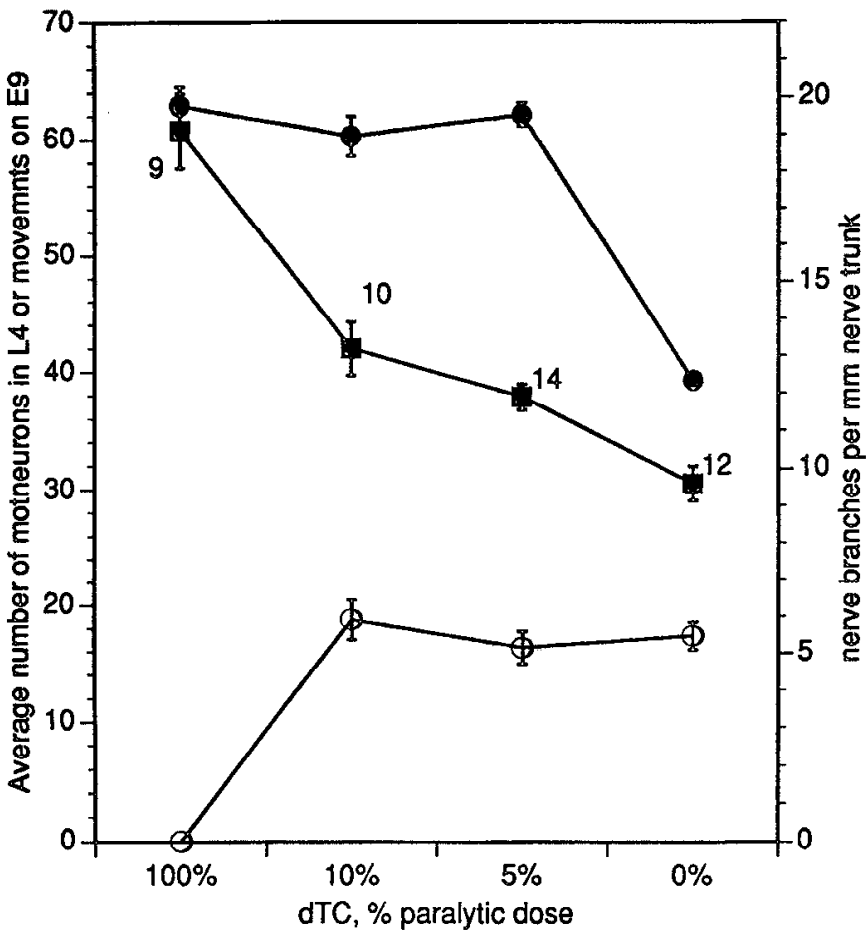

Figure 6. Effects of dTC treatments on side branch formation of intramuscular nerve trunks in the slow anterior region of the iliofibularis muscle. Branch formation is indicated as the average number of branches per mm of nerve trunk ( $\square$ ). The doses of dTC on the horizontal axis indicate percentages of the daily paralytic dose, as in Figure 3 . The effects of these doses on motoneuron survival $(\mathbf{)}$ ) and embryonic motility $(\mathrm{O})$, determined in earlier experiments, are given for comparison (refer to Fig. 3). The smallest dose of dTC that still had a maximal effect on motoneuron survival (5\% of the paralytic dose) resulted in a small but significant increase in nerve branches per millimeter of nerve trunk $(p<0.05)$, as did the $10 \%$ dose $(p<0.01)$. However, both subparalytic doses resulted in a significantly lower increase in branch formation than the paralytic dose $(p<0.01)$.

or motoneuron survival. As illustrated in Figure 6, the smallest dose of dTC that still had a maximal effect on motoneuron survival $(5 \%)$ resulted in a small but significant increase from 9.6 \pm 0.5 to $11.9 \pm 0.4$ branches per mm of nerve trunk $(p<0.05)$. A $10 \%$ dose increased side branch number significantly to 13.2 \pm 0.7 per mm of nerve trunk $(p<0.01)$. However, both subparalytic doses resulted in a significantly lower increase in branch formation than the paralytic dose $(p<0.01)$.

These results show that although subparalytic dTC treatments do increase the number of intramuscular nerve branches, significantly higher concentrations are required for a maximal effect than are necessary to block naturally occurring motoneuron death. One interpretation of these results is that increased branch formation and increased motoneuron survival may be two independent consequences of drug treatment, and that the former is not directly responsible for the latter. Alternatively, a small increase in side branch formation might be sufficient to rescue all motoneurons, further increases having no effect simply because all motoneurons already survive.

\section{Discussion}

Previous studies have shown that essentially all motoneurons generated in the spinal cord survive if a paralytic dose of either $\alpha$-BTX or dTC is applied to developing chick embryos through- out the period of naturally occurring motoneuron death (Pittman and Oppenheim, 1978; Pittman and Oppenheim, 1979). We have investigated the effects of decreasing doses of $\alpha$-BTX and dTC on both embryonic motility and motoneuron survival, to determine if neuromuscular blockade is a necessary component of the toxin-induced arrest of naturally occurring motoneuron death. Subparalytic doses of both dTC and $\alpha$-BTX are sufficient to rescue essentially all motoneurons despite having no observable effect on embryonic motility. The different dose-response curves suggest that the binding site(s) for these drugs that are responsible for increasing motoneuron survival may not be nAChRs at neuromuscular junctions. Neuronal nAChRs seem a likely alternative. The $\alpha$-BTX binding site identified in the spinal cord by Renshaw and coworkers (1993) is a possible candidate, although there is no direct evidence for this idea. At least three chick neuronal nAChRs are known to bind $\alpha$-BTX (Anand et al., 1993; Keyser et al., 1993). Whether the spinal cord binding site corresponds to one of these three is unknown, as is whether the spinal site is actually involved in the effects of the cholincrgic drugs on motoneuron survival.

To determine if nicotinic cholinergic receptor blockade could influence motoneuron survival in the absence of muscle type $\mathrm{nAChRs}$, the effect of dTC on motoneuron survival in vitro was also investigated. Addition of dTC to mixed spinal cord cultures plated with muscle extract increased motoneuronal survival. Muscle cells were not present in these cultures. This indicates that UTC influenced survival by acting directly on spinal cord cells, through some type of $\mathrm{nAChR}$ present on motoneuronal cell bodies and/or their growing processes, or even on some other cell type in the spinal cord. This is consistent with a previous report indicating the developmental appearance of an $\alpha$-BTX binding site on rodent spinal cord neurons in culture (Schaffner and Olek, 1985). These results provide additional support for the idea that muscle type nAChRs may not mediate toxin-induced arrest of naturally occurring motoneuron death in vivo, although we cannot rule out the possibility that the actions of the drug in vivo and in vitro may not be the same.

In culture, dTC potentiated the survival effects of muscle extract rather than affecting motoneuronal survival independently. The drug also did not protect motoneurons in established cultures from degeneration following the withdrawal of muscle extract. Similarly, in the limb ablation studies, neither dTC nor $\alpha$-BTX could protect motoneurons from death in the absence of target. A straightforward interpretation of these results is that dTC and $\alpha$-BTX may modify the response of motoneurons to the target-derived factors necessary for their survival, but that the blockade of neuronal $\mathrm{nAChRs}$ is not sufficient by itself to protect these cells from death.

These results place important restrictions on the possible mechanisms responsible for the survival-promoting effects of nicotinic antagonists. Neither neuromuscular blockade nor a reduction in the activity of motoneurons is likely to be critically involved. An effect of nicotinic blockers on motoneuronal activity would also be expected to result in decreased embryonic motility, because movement is neurally induced (Landmesser and Morris, 1975). The present results also argue against another proposed explanation of how dTC might increase motoneuronal survival. Because dTC and $\alpha$-BTX act directly on the spinal cord to dccrcasc motoncuronal activity (Landmesser and Szente, 1986), Tang and Landmesser (1993) suggested that less active motoneurons may require less trophic factor. Given a limited amount of factor, more motoneurons could survive if each one 
required less factor. This explanation is now unlikely, however, because overall motoneuronal activity in treated animals need not be appreciably affected.

Although all motoneurons generated in the spinal cord survive if a paralytic dose of either $\alpha$-BTX or dTC is applied to developing chick embryos throughout the period of naturally occurring motoneuron death (Pittman and Oppenheim, 1978; Pittman and Oppenheim, 1979), muscle fibers do not increase trophic factor production in response to neuromuscular blockade (Tanaka, 1987; Houenou et al., 1991). A variation of the neurotrophic hypothesis for motoneurons could therefore be that muscle fibers normally produce enough trophic factor for all motoneurons, but that access to this factor is limited. Neuromuscular blockade might increase motoneuron access to trophic factor (Oppenheim, 1989; Dahm and Landmesser, 1991). A correlation between increased motoneuron survival and increased intramuscular nerve branching, as a result of paralytic treatment with dTC or $\alpha$-BTX, has been previously established (Dahm and Landmesser, 1988). Treatment results in the defasiculation of growing intramuscular nerves and greatly increases the number of nerve branches that form within muscles. An increase in branching might increase motoneuron access to trophic factor.

To examine the correlation between increased branch formation and increased motoneuron survival more completely, we studied the effects of subparalytic doses of dTC on branch formation. These doses resulted in small but significant increases in branch number. If increased intramuscular nerve branch formation is necessary for dTC to influence motoneuron survival, then a minimal increase in branch formation is sufficient for a maximal effect on motoneuron survival. No cause-and-effect relationship between branch formation and survival has yet been established, however, so the two may also be independent consequences of nicotinic blockade. Furthermore, small increases in branching were observed without changes in motility. This suggests that the effect of dTC on branching may also not be mediated by blocking muscle type nAChRs.

As increased nerve branching is correlated with increased survival, so a reduction of intramuscular nerve branching and synaptogenesis is correlated with decreased motoneuron survival (Tang and Landmesser, 1993). Treatment of chick embryos with an endoneuramidase specific for polysialic acid throughout the period of naturally occurring motoneuron death, resulted in reduced intramuscular nerve branching and diminishẹd motoneuron survival with no affect on neuronal or muscle cell activity. This result strengthens the correlation between the ability of motoneurons to survive and the extent to which they branch within muscles and consequently form synapses. Some caution may be necessary, however. If the adhesive interactions between axons and myotubes are altered, it could result in decreased motoneuron survival without specifically affecting the process of naturally occurring death. Furthermore, decreased intramuscular nerve branching could decrease the access of motoneurons to trophic factor and potentiate cell death, without necessarily implying that increased branching is required for the increased motoneuron survival observed in embryos treated with cholinergic blockers.

Synaptic transmission between neurons remains the best characterized function of neurotransmitters, but these molecules also regulate longer term changes in the cellular properties of neurons and other postsynaptic cells. Neuronal nAChRs generally permit $\mathrm{Ca}^{2+}$ influx when activated. The physiological consequences of this influx include dendritic retraction in chick ciliary ganglion neurons (Pugh and Berg, 1994), effects on the GABA response of neurons in the rat medial habenular nucleus (Mulle et al., 1992), and growth cone orientation in embryonic Xenopus spinal cord neurons (Zheng et al., 1994). It is possible that dTC and $\alpha$-BTX might save motoneurons by blocking a neuronal $\mathrm{nAChR}$ and thereby reducing $\mathrm{Ca}^{2+}$ entry. Intracellular $\mathrm{Ca}^{2+}$ levels are one factor influencing neuronal survival, and $\mathrm{Ca}^{2+}$ influx through NMDA receptors is linked to excitotoxic neuronal death in the mature nervous system (Gibbons et al., 1993). The parameters governing motoneuronal survival during development are only partially understood, however, and both the location of any neuronal nAChRs involved in this process and the precise nature of their involvement remain to be determined.

\section{References}

Anand R, Peng X, Ballesta JJ, Lindstrom J (1993) Pharmacological characterization of alpha-bungarotoxin sensitive acetylcholine receptors immunoisolated from chick retina: contrasting properties of $\alpha 7$ and $\alpha 8$ subunit-containing types. Mol Pharmacol 44:1046-1050.

Dahm L, Landmesser L (1988) The regulation of intramuscular nerve branching during normal activity and following activity blockade. Dev Biol 130:621-644.

Dahm L, Landmesser L (1991) The regulation of synaptogenesis dur ing normal activity and following activity blockade. J Neurosci 11: 238-255.

Davies AM (1986) The survival and growth of embryonic proprioceptive neurones is promoted by a factor present in skeletal muscle. Dev Biol 115:56-67.

Hamburger V (1948) The mitotic patterns in the spinal cord of the chick embryo and their relation to histogenic processes. J Comp Neurol 88:221-284.

Hamburger V (1975) Cell death in the development of the lateral motor column of the chick embryo. J Comp Neurol 160:535-546.

Hory-Lee F, Russell M, Lindsay RM, Frank E (1993) Neurotrophin 3 supports the survival of developing muscle sensory neurons in culture. Proc Natl Acad Sci USA 90:2613-2617.

Ilouenou LJ, McManaman JL, Prevette D, Oppenheim RW (1991) Regulation of putative muscle-derived neurotrophic factors by muscle activity and innervation: in vivo and in vitro studies. J Neurosci 11: $2829-2837$.

Keyser KT, Britto LRG, Schoepfer R, Whiting P, Cooper J, Conroy W, Brozozowska-Prechtl A, Karten HJ, Lindstrom J (1993) Three types of $\alpha$-bungarotoxin-sensitive nicotinic acetylcholine receptors are expressed in chick retina. J Neurosci 13:442-454.

Landmesser $\mathrm{L}$ (1978) The development of motor projection patterns in the chick hind limb. J Physiol (Lond) 284:391-414.

Landmesser L, Morris D (1975) The development of functional innervation in the hind limb of the chick embryo. J Physiol (Lond) 249: $301-326$.

Landmesser LT, Szente M (1986) Activation patterns of embryonic chick hindlimb muscles following blockade of activity and motoneurone cell death. J Physiol (Lond) 380:157-174.

Lindstrom J, Schoepfer R, Conroy W, Whiting P, Das M, Saedi M, Anand R (1991) The nicotinic acetylcholine receptor gene family: structure of nicotinic receptors from muscle and neurons and neuronal $\alpha$-bungarotoxin-binding proteins. Adv Exp Med Biol 287:255-278.

Mulle C, Choquet D, Korn H, Changeux JP (1992) Calcium influx through nicotinic receptors in rat central neurons: its relevance to cellular regulation. Neuron 8:135-143.

Oppenheim RW (1989) The neurotrophic theory and naturally occurring motoneuron death. Trends Neurosci 12:252-255.

Oppenheim RW, Prevette D, Haverkamp LJ, Houenou L, Yin QW, McManaman J (1993) Biological studies of a putative avian musclederived neurotrophic factor that prevents naturally occurring motoneuron death in vivo. J Neurobiol 24:1065-1079.

Pittman R, Oppenheim RW (1978) Neuromuscular blockade increases motoneuron survival during normal cell death in the chick embryo. Nature 271:364-366.

Pittman R, Oppenheim RW (1979) Cell death of motoneurons in the chick embryo spinal cord. IV. Evidence that a functional neuromuscular interaction is involved in the regulation of naturally occurring 
cell death and the stabilization by synapses. J Comp Neurol 187:425446.

Pugh PC, Berg DK (1994) Neuronal acetylcholine receptors that bind $\alpha$ - bungarotoxin mediate neurite retraction in a calcium-dependent manner. J Neurosci 14:889-896.

Renshaw G, Rigby P, Self G, Lamb A, Goldie R (1993) Exogenously administered $\alpha$-bungarotoxin binds to embryonic spinal cord: implications for the toxin-induced arrest of naturally occurring motoneuron death. Neuroscience 53:1163-1172.

Schaffner AE, Olek AJ (1985) The developmental appcarance of $\alpha$ bungarotoxin binding sites on rodent spinal cord neurons in culture. Dev Brain Res 25:239-247.
Tanaka H (1987) Chronic application of curare does not increase the level of motoneuron survival-promoting activity in limb muscle extracts during the naturally occurring motoneuron cell death period. Dev Biol 124:347-357.

Tang J, Landmesser L (1993) Reduction of intramuscular nerve branching and synaptogenesis is correlated with decreased motoneuron survival. J Neurosci 13:3095-3103.

Vijayaraghavan S, Pugh PC, Zhang ZW, Rathouz MM, Berg DK (1992) Nicotinic receptors that bind $\alpha$-bungarotoxin on neurons raise intracellular $\mathrm{Ca}^{2+}$. Neuron 8:353-362.

Williams C, Wohlenberg G, O'Donovan MJ (1987) Regional variations in the extent and timing of motoneuron death in the lumbosacral spinal cord of the chick embryo. Brain Res 34:215-221. 